\title{
Quetiapine, an Atypical Antipsychotic, Is Protective against Autoimmune-Mediated Demyelination by Inhibiting Effector T Cell Proliferation
}

\author{
Feng $\mathrm{Mei}^{19}$, Sheng Guo ${ }^{29}$, Yangtao $\mathrm{He}^{1}$, Linyun Wang ${ }^{1}$, Hongkai Wang ${ }^{1}$, Jianqin Niu' ${ }^{1}$, Jiming Kong ${ }^{3}$, \\ Xinmin $\mathrm{Li}^{4}$, Yuzhang $\mathrm{Wu}^{2}$, Lan $\mathrm{Xiao}^{1 *}$
}

1 Department of Histology and Embryology, Chongqing Key Laboratory of Neurobiology, Third Military Medical University, Chongqing, China, 2 Department of Immunology, Third Military Medical University, Chongqing, China, 3 Department of Human Anatomy and Cell Science, University of Manitoba, Winnipeg, Canada, 4 Department of Psychiatry, University of Manitoba, Winnipeg, Canada

\begin{abstract}
Quetiapine (Que), a commonly used atypical antipsychotic drug (APD), can prevent myelin from breakdown without immune attack. Multiple sclerosisis (MS), an autoimmune reactive inflammation demyelinating disease, is triggered by activated myelin-specific T lymphocytes (T cells). In this study, we investigated the potential efficacy of Que as an immunemodulating therapeutic agent for experimental autoimmune encephalomyelitis (EAE), a mouse model for MS. Que treatment was initiated on the onset of $\mathrm{MOG}_{35-55}$ peptide induced EAE mice and the efficacy of Que on modulating the immune response was determined by Flow Cytometry through analyzing $\mathrm{CD} 4^{+} / \mathrm{CD}^{+}$populations and the proliferation of effector T cells $\left(C D 4^{+} C D 25^{-}\right)$in peripheral immune organs. Our results show that Que dramatically attenuates the severity of EAE symptoms. Que treatment decreases the extent of $C D 4^{+} / C D 8^{+} T$ cell infiltration into the spinal cord and suppresses local glial activation, thereby diminishing the loss of mature oligodendrocytes and myelin breakdown in the spinal cord of EAE mice. Our results further demonstrate that Que treatment decreases the $\mathrm{CD}^{+} / \mathrm{CD}^{+} \mathrm{T}$ cell populations in lymph nodes and spleens of EAE mice and inhibits either $\mathrm{MOG}_{35-55}$ or anti-CD3 induced proliferation as well as IL-2 production of effector T cells $\left(C D 4^{+} C D 25^{-}\right)$isolated from EAE mice spleen. Together, these findings suggest that Que displays an immunemodulating role during the course of EAE, and thus may be a promising candidate for treatment of MS.
\end{abstract}

Citation: Mei F, Guo S, He Y, Wang L, Wang H, et al. (2012) Quetiapine, an Atypical Antipsychotic, Is Protective against Autoimmune-Mediated Demyelination by Inhibiting Effector T Cell Proliferation. PLoS ONE 7(8): e42746. doi:10.1371/journal.pone.0042746

Editor: Yue Feng, Emory University, United States of America

Received May 24, 2012; Accepted July 12, 2012; Published August 13, 2012

Copyright: $\odot 2012$ Mei et al. This is an open-access article distributed under the terms of the Creative Commons Attribution License, which permits unrestricted use, distribution, and reproduction in any medium, provided the original author and source are credited.

Funding: This work is in part supported by the National Natural Science Foundation of China (NSCF, 81071084), International Science \& Technology Cooperation Program of China (2010DFB30820) and Natural Science Foundation Project of Chongqing (CQ CSTC, 2009BB5157, 2010BB5157). The funders had no role in study design, data collection and analysis, decision to publish, or preparation of the manuscript.

Competing Interests: The authors have declared that no competing interests exist.

* E-mail: xiaolan35@hotmail.com

9 These authors contributed equally to this work.

\section{Introduction}

Disseminated demyelination in the central nervous system (CNS) mediated by autoimmune reactive inflammation is the primary pathological hallmark in multiple sclerosis (MS) and in various animal models, including experimental autoimmune encephalomyelitis (EAE), which results in axonal injury, synaptic dysfunctions and neurological impairments $[1,2]$. The activated myelin-specific $\mathrm{CD}^{+}$lymphocytes ( $\mathrm{T}$ cells) that infiltrated into CNS nerve tissue have been considered as the initiator or early effector cells in the development of both EAE and MS [3,4].

For therapy of MS or EAE, a series of strategies including immunoregulation, anti-inflammation and enhancement in neuroprotection and/or neuroregeneration mediated by small molecules or mesenchymal stem cells have been applied to re-balance/control the immune response and protect myelin from breakdown [5-9]. Currently, interferon $\beta$ (IFN $\beta$ ) and glatiramer acetate (GA) are commonly used to treat MS [10,11]. These drugs act mainly to rebalance the immune response and are capable of slowing down disease progress and ameliorating the frequency of recurrence of
MS, but the efficacy is still limited and the long-term outcome is not satisfactory, most likely due to the inefficiency in myelin repair and neuronal degeneration [1]. Therefore, to rescue myelin from breakdown and to promote remyelination should be incorporated into the approach and treatment of demyelinating diseases including MS.

Quetiapine (Que) is a commonly used atypical antipsychotic drug (APD) that has superior therapeutic effects on negative and cognitive symptoms in patients with schizophrenia and other neurological disorders like depression. Previous in vivo and in vitro studies have demonstrated that Que exerts protective effects on neurons [12]. Recently, oligodendrocyte dysfunction or demyelination has been implicated in the pathophysiology of schizophrenia, bipolar disorder and major depression [13]. In trying to reveal the cellular mechanisms underlying the therapeutic actions of Que, recent studies indicate that Que can effectively prevent myelin breakdown in the cerebral cortex and the concomitant spatial working memory impairment in cuprizone induced demyelination mouse model without immune attacks [14-16]. Furthermore, Que can inhibit the activation of microglia in the 
brain of these mice $[16,17]$. These observations suggest that Que may address two key aspects involved in the pathophysiological process of MS, namely prevention of demyelination and modulation of the local glial activation, which suggests broader potentials for Que in demyelination diseases. However, it is still unknown whether Que displays an immune-modulating role and may be a promising candidate for treatment of MS.

To investigate the potential efficacy of Que as a therapeutic agent, we utilized the MOG induced EAE mouse model to mimic MS. We demonstrate that Que dramatically attenuates the severity of EAE symptoms, diminishes demyelination and the infiltration of $\mathrm{CD}^{+} / \mathrm{CD}^{+} \mathrm{T}$ cells, as well as activation of local microglia in the spinal cord. Additionally, our results indicate that Que exerts immunomodulatory capacities to attenuate $\mathrm{MOG}_{35}$ $55^{-s p e c i f i c ~ i m m u n e ~ r e s p o n s e, ~ a n d ~ t o ~ i n h i b i t ~ e f f e c t o r ~} \mathrm{~T}$ cell proliferation and thus reduce peripheral $\mathrm{CD} 4^{+} / \mathrm{CD}^{+} \mathrm{T}$ population as administrated to EAE mice. Overall, our findings demonstrate the utility of Que as a potential therapeutic agent for demyelinating diseases such as MS.

\section{Materials and Methods}

\section{EAE Mice Model and Treatment Protocols}

C57BL/6 mice were obtained from the Animal Center of Third Military Medical University. All experiments were performed in accordance with Health Guide for the Care and Use of Laboratory Animals, with the approval of Third Military Medical University Committee on Animal Care (permission NO: SCXKJUN-2007-015). Female C57BL/6 mice ( $N=43$, 8 weeks old) were immunized subcutaneously with $200 \mu \mathrm{g}$ of $\mathrm{MOG}_{35-55}$ peptide (Invitrogen, Carlsbad, CA) emulsified in complete Freund adjuvant (CFA, Sigma Aldrich, Saint Louris, MO) on Day 0 and Day 7, and received $300 \mathrm{ng}$ pertussis toxin (PT, List Biological Laboratories, Campbell, CA) in $0.1 \mathrm{ml}$ PBS intraperitoneal at the time of immunization and 48 hours later. The control $(\mathrm{N}=10)$ mice were immunized with bovine serum albumin (BSA) at the same dosage $(200 \mu \mathrm{g})$ followed by PT and the unimmunized mice $(\mathrm{N}=9)$ were given only PBS and PT. Onset and clinical scores of EAE symptoms were evaluated daily using a neurological score as follows: 0 , no clinical signs; 0.5 , partially limp tail; 1 , paralyzed tail; 2 , loss in coordinated movement; hind limb paresis; 2.5 , one hind limb paralyzed; 3, both hind limbs paralyzed; 3.5, hind limbs paralyzed with weakness in forelimbs; 4, forelimbs paralyzed; 5, moribund as previously described [18].

\section{Quetiapine Treatment Protocols}

Quetiapine (AstraZeneca, Wilmington, DE) (dissolved in distilled water) was orally administrated to the mice $(10 \mathrm{mg} / \mathrm{kg}$ / day) [14]. Que was administrated orally in EAE groups (Que treated) on Day 16 after immunization and maintained for 24 days to test clinical symptoms (40 days after immunization) and histology changes were detected on 30 days after immunization, a time at which apparent histopathology changes can be detected, while those in the untreated groups were given only distilled water. To test immunoresponse in periphery immune organs, Que was orally administrated 1 week before immunization, and mice were sacrificed on Day 10 after immunization, a time at which very efficient $\mathrm{MOG}_{35-55}$-specific responses can be detected.

\section{Immunocytochemical Staining and Quantification}

On Day 30 after immunization, mice were deeply anesthetized with $1 \%$ pentobarbital and transcardially perfused with $4 \%$ paraformaldehyde in PBS. Spinal cords were dehydrated in $30 \%$ sucrose and crossly cut $(20 \mu \mathrm{m})$ using a cryostate microtome (MS 1900, Leica). Sections were blocked with 10\% BSA and incubated with rat anti-CD4, CD8, CD68, CD $11 \mathrm{~b}$ antibodies, or goat anti-MBP, rabbit anti-NG2, mouse anti-APC, and mouse anti-GFAP antibodies (Table S1) overnight at $4^{\circ} \mathrm{C}$ followed by using an Alex Fluor 488, 568 or Cy5-conjugated secondary antibody relatively. The results were examined under a fluorescence microscope (90 i, Nikon,) or a laser confocal scanning microscope (PV100, Olympus) with the excitation wavelengths

A

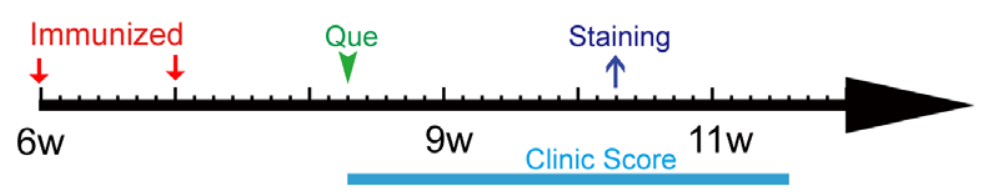

\section{B}

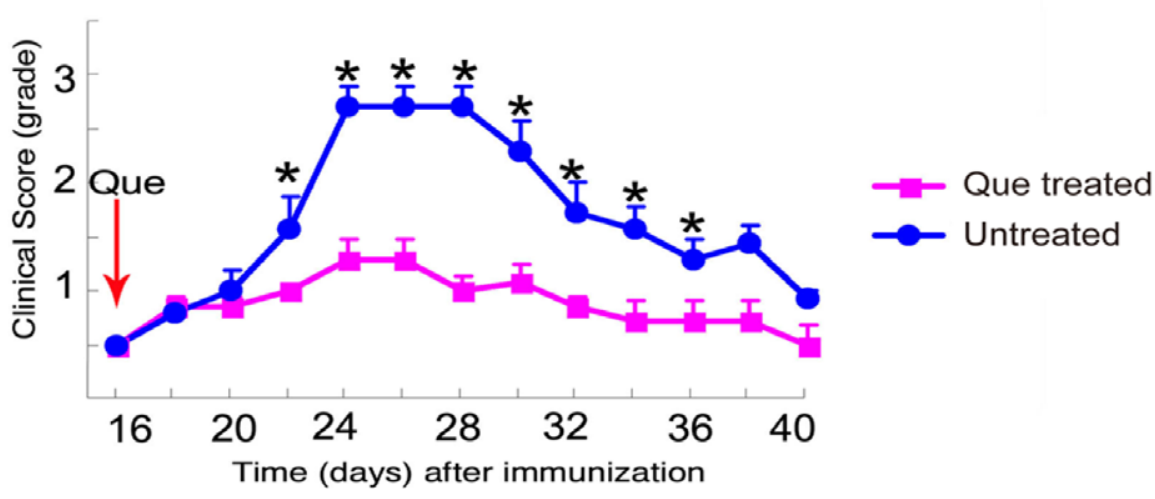

Figure 1. Post-treatment of Que rescues EAE mice from deterioration. A: Schematic diagram displaying the time course of immunization and Que post-treatment. B: Que post-treatment was initiated on day 16 post-immunization when mice attained a clinical score of 0.5 (arrow). Untreated mice $(\mathrm{N}=9)$ continued to deteriorate with increasing clinical scores that reached values of approximately 3 . Mice treated with Que $(\mathrm{N}=9)$ with an initial score of 0.5 reached a value just greater than 1 , and were stabilized and maintained at that value. Values shown are means \pm SEM $(*, p<0.05)$.

doi:10.1371/journal.pone.0042746.g001 


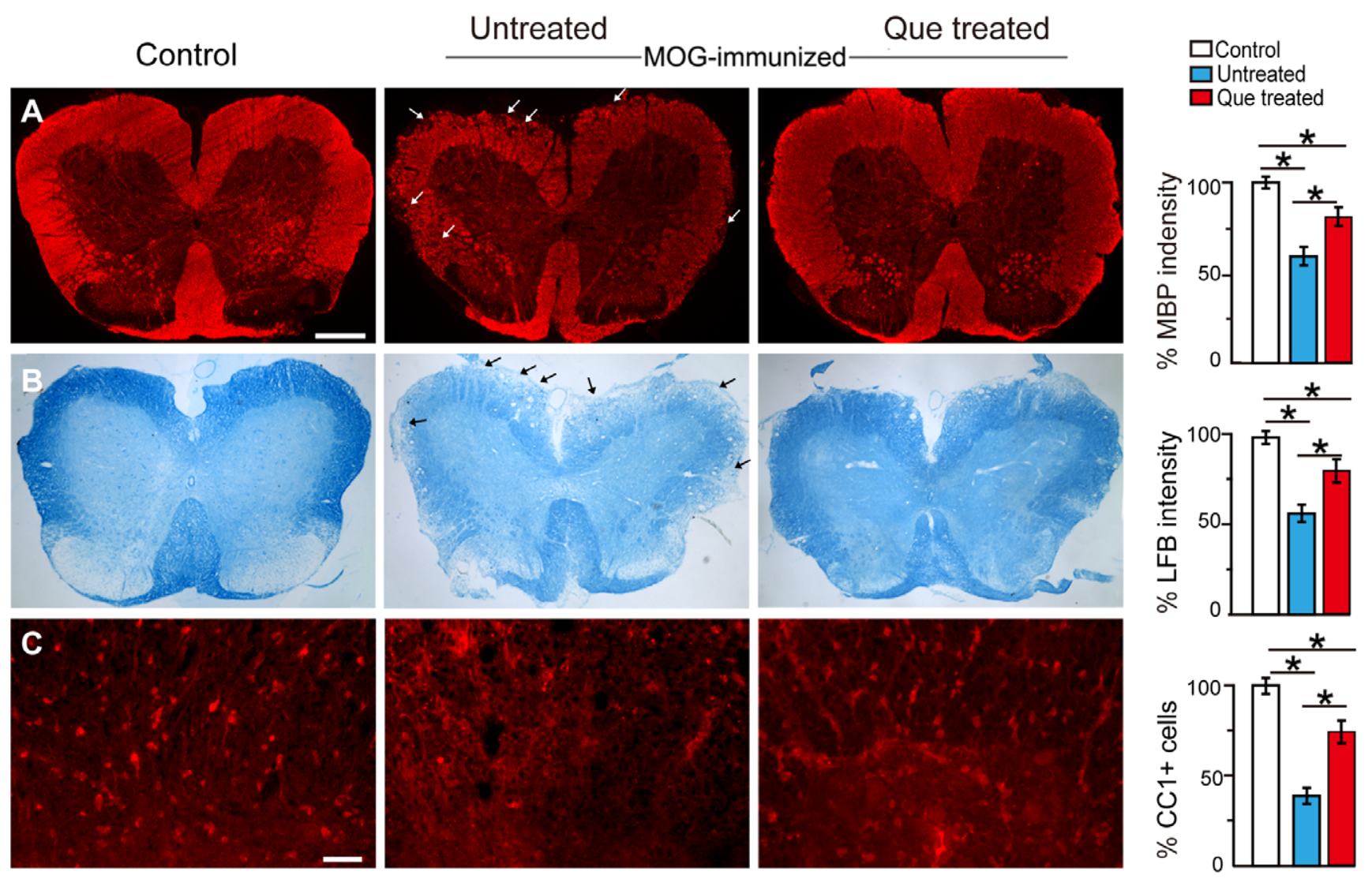

Figure 2. Que protectes the spinal cord from demyelination and loss of oligodendrocytes. A. MBP immunofluorescent staining displays an obvious decrease of myelin (arrows) in the white matter of the spinal cord in the untreated EAE controls $(N=5)$. Post-treatment with Que $(N=5)$ protects myelin from breakdown and displays a similar fluorescence intensity to the unimmunized controls $(N=5)$. B. Luxol Fast Blue $(L F B)$ staining indicates a similar pattern after Que treatment (arrows). C. Que prevents the loss of $\mathrm{CC}^{+}$oligodendrocytes as compared with the untreated controls. Quantification of the observations is provided in the bar graphs. $\left({ }^{*}, p<0.05\right)$. Scale bar $A=B=0.5 \mathrm{~mm}, C=0.2 \mathrm{~mm}$. doi:10.1371/journal.pone.0042746.g002

proper for Alex Fluor 488 (488 nm), Alex Fluor 568 (568 nm), Cy5 $(628 \mathrm{~nm})$ or DAPI $(380 \mathrm{~nm})$. For stereological quantification, serial sections of spinal cord from L1-L6 were collected (about 200 sections) and twenty sections were sampled from each animal in a systematic and random manner. In practice, every first and the tenth sections were sampled from the 200 sections systematically. After immunofluorescence staining, digital images of CD4+, CD8+, CC1+, NG2+, GFAP+, CD8+, CD11b+, CD68+ and MBP staining were acquired with a digital camera (Nikon, Japan) mounted on a 90 i fluorescence microscope (Nikon, Japan). The cell numbers of CD4, CD8, CG1 and NG2 positive cells and the mean intensity of CD1 lb+, GFAP+, CD68+, MBP+ and Fast Blue staining were quantified with Image-Pro Plus 5.0 (Media Cybernetics, Silver Spring, MD, USA).

\section{Flow Cytometry Analysis}

Mice were anesthetized, spleen and draining lymph nodes removed, and single cell suspension was prepared. Cells isolated from lymph nodes and spleens were adjusted at $1 \times 10^{6} \mathrm{cells} / \mathrm{vial}$ and stained with Percp cy5.5-anti-CD4(clone RM4-5), PE-antiCD25(clone PC61.5) or Percp cy5.5-anti-GD8 (clone 53-6.7,) for $30 \mathrm{~min}$ at $4^{\circ} \mathrm{C}$, then washed with PBS containing $1 \%$ FCS. All antibodies were obtained from eBioscience. In some instances, isotype-matched IgG were used as negative controls. In each test, 1,000,000 cells were collected by a Canto II flow cytomerer using
Cell Quest Diva software (BD Biosciences) and analyzed by FlowJo software (TriStar).

\section{CFSE Proliferation Assay}

Proliferation of T cells was measured by dilution of the dye CFSE.

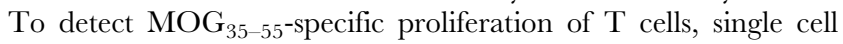
suspensions from spleen were obtained at 10 days after $\mathrm{MOG}_{35-55^{-}}$ immunization. Cells were incubated with CFSE $(2 \mu \mathrm{M})$ (eBioscience) for $10 \mathrm{~min}$ at $37^{\circ} \mathrm{C}$ in the dark, then washed with cold complete media 3 times. Cells labeled with CFSE were cultured in 96-well plates $\left(2 \times 10^{5}\right.$ cells/well $)$ and stimulated with indicated concentration of $\mathrm{MOG}_{35-55}$ peptide in triplicate at $0,1,10$ or $100 \mu \mathrm{g} / \mathrm{ml}$. In additional experiments, $\mathrm{CD} 4^{+} \mathrm{CD} 25^{-} \mathrm{T}$ cells in spleens of naïve $\mathrm{C} 57 \mathrm{BL} / 6$ mice $(\mathrm{N}=10)$ were fractionated using magnetic bead chromatography (Miltenyi). Purity of the samples was routinely tested after sorting and was $>95 \%$. Cells were the labeled with CFSE and cultured in 96-well plates $\left(2 \times 10^{5}\right.$ cells/well $)$ coated with anti-CD3 antibody $(1 \mu \mathrm{g} / \mathrm{ml})$ by adding Que $(1 \mu \mathrm{M})$ or not for $72 \mathrm{~h}$. The results were analyzed using FACS (Canto II, BD).

\section{RNA Preparation and Real-time PCR}

$\mathrm{CD} 4^{+} \mathrm{CD} 25^{-} \mathrm{T}$ cells were cultured in 96-well plate coated with anti-CD3 $(1 \mu \mathrm{g} / \mathrm{ml})$, incubating with/wihout Que $(1 \mu \mathrm{M})$ for different time $(0,12,24$ or $48 \mathrm{~h})$, total RNA was extracted with Trizol Reagent (Invitrogen) following the manufacture's instructions after indicated time. Reverse transcription of RNA was performed using 


\section{Control}
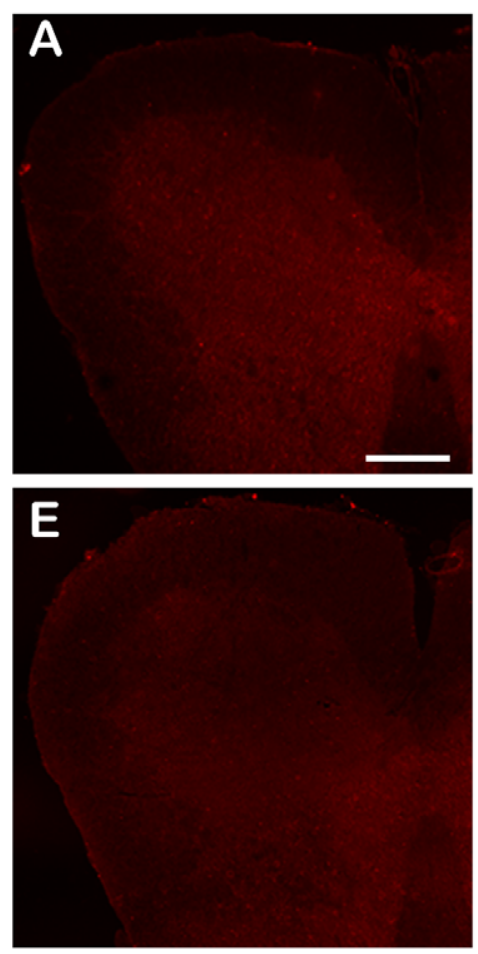

Untreated
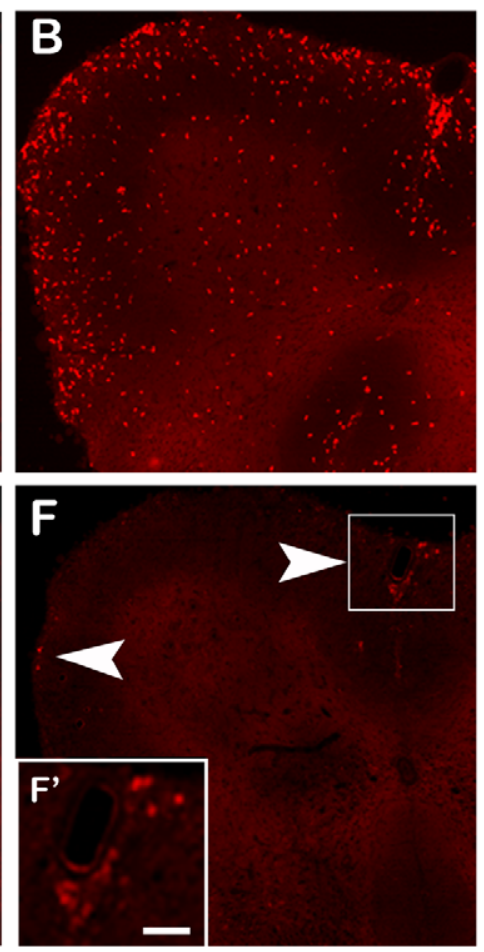

Que treated
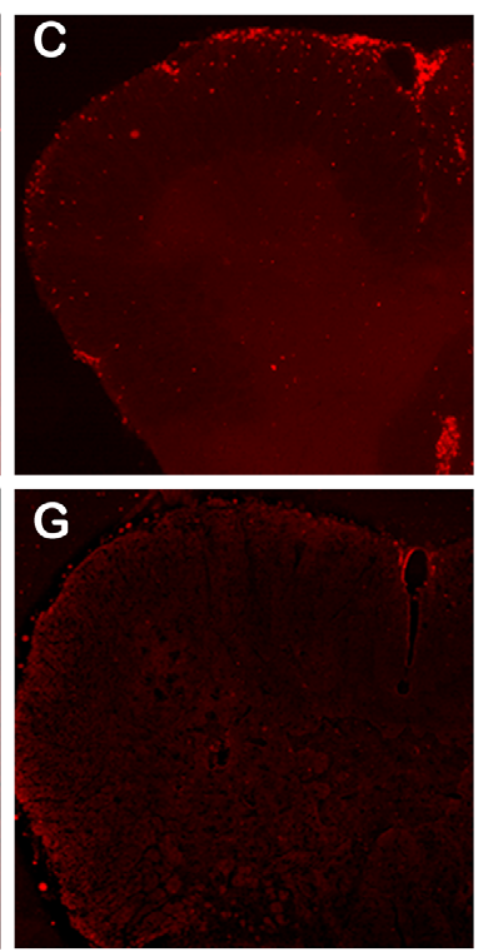
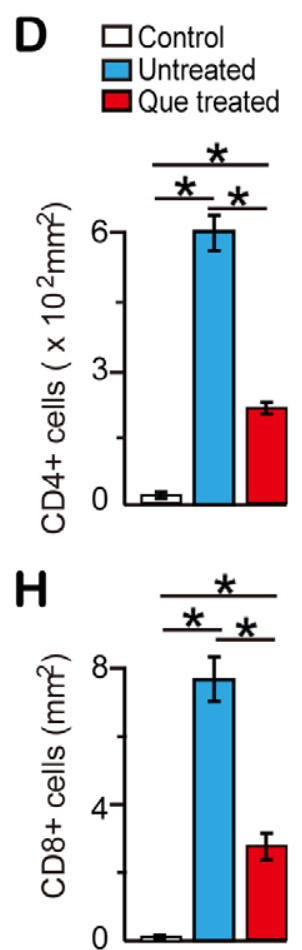

Figure 3. Que decreases the number of infiltrating $\mathbf{T}$ cells in spinal cord. Panels display the number of infiltrating CD4/8 positive $T$ cells in the EAE spinal cord with/without Que treatment. A-C: $C D 4^{+}$cells were not observed in the spinal cord prior to immunization $(\mathbf{A})$. An extensive infiltration of $\mathrm{CD}^{+}$cells was detected throughout the spinal cord and enriched in the white matter of the EAE mice (B). Que treatment significantly decreases the amount of infiltrating $\mathrm{CD}^{+}{ }^{\mathrm{T}}$ cells $(\mathbf{C})\left(\mathrm{N}=5,{ }^{*}, P<0.05\right)$. E-G: $\mathrm{CD} 8^{+}$cells display a similar decrease after Que treatment $(\mathrm{N}=5$, ${ }^{*}, p<0.05$ ), but the overall extent of $\mathrm{CD}^{+}$cells detected was much less than that of $\mathrm{CD} 4^{+}$cells. Quantification of the staining is depicted in the bar graphs (D and $\mathbf{H}$ ). Scale bar $\mathbf{A}-\mathbf{G}=0.5 \mathrm{~mm}, \mathbf{F}^{\prime}=50 \mu \mathrm{m}$.

doi:10.1371/journal.pone.0042746.g003

an RNA PCR Kit (AMV) (Takara). The cDNA was analyzed by realtime PCR with the Rotor Gene6000 (Corbett Research, Australia) according to the protocol provided by the manufacturer and $2^{-\Delta \Delta \mathrm{Ct}}$ method. Briefly, PGRs were performed using SYBR premix Ex Taq (Takara) in a final volume of $20 \mu \mathrm{l}$. The thermal conditions were $95^{\circ} \mathrm{C}$ for 10 seconds followed by 40 cycles of $95^{\circ} \mathrm{C}$ for 5 seconds, $60^{\circ} \mathrm{C}$ for 15 seconds and $72^{\circ} \mathrm{C}$ for 15 seconds. The primers were IL-2 sense $5^{\prime}-$ CATTGACACTTGTGCTCGTTG-3', antisense $\quad 5^{\prime}-$ GGTTCGTGTAATTCTCCATCCTG-3', mouse actin- $\beta$ sense $5^{\prime}$-CGTGCGTGACATTAAGGAGAAG- ${ }^{\prime}$, antisense $5^{\prime}$ GGAAGGAAGGGTGGAAGAGTG-3'.

\section{Detection of IL-2 by ELISA}

The supernatants were obtained and examined by ELISA (eBioscience). In brief, serially diluted supernatants samples and internal standards samples (recombinant murine IL-2) were incubated with immobilized antibody. Antibody was detected with HRP-labeled rabbit anti-mouse IgG1 and IgG2a (Zymed Laboratories), which were then detected with the substrate o-phenylenediamine. The relative concentration of antibody was determined from a standard curve of known concentrations of unlabeled murine IL-2 antibody (Southern Biotechnology Associates).

\section{Statistical Analysis}

Data were expressed as means \pm SEM. Statistical analysis between experimental groups was evaluated using analysis of variance (ANOVA). A two-tailed paired Student's t test was used for comparing individual treatment groups. A probability of $p<0.05$ was taken as a statistically significant difference.

\section{Results}

Que Administration Attenuates Clinical Signs and Protects Myelin from Breakdown in EAE

Que treatment was initiated on day 16 post-immunization when mice attained a clinical score of 0.5 , and mice were monitored for a total of 40 days (Fig. 1A). The untreated EAE mice continued to deteriorate with increased clinical scores that reached a value of approximately 3, whereas mice treated with Que reached a value of 1 , then stabilized at that level and did not display further deterioration (Fig. 1B). While unimmunized group and control (immunized with BSA) mice displayed normal behavior (data not shown). Therefore, Que treatment clearly attenuates the clinical scores in EAE. Additionally, pre-treatment with Que from 1 week before immunization resulted in a significant delay in the onset of $\mathrm{EAE}$ and diminishes the severity of symptoms (Figure $\mathrm{S} 1$ ).

As demyelination is one of the major histopathologic hallmarks in EAE and MS, histological observation indicate that the spinal cords from the immunized group without Que treatment were weakly stained with MBP and LFB, especially in the white matter tracts (arrows, Fig. 2A, B), while a more intense expression of MBP and LFB staining were observed in the Que treatment group but less intense than unimmunized group or control (Fig. 2A, B) on 30 
A
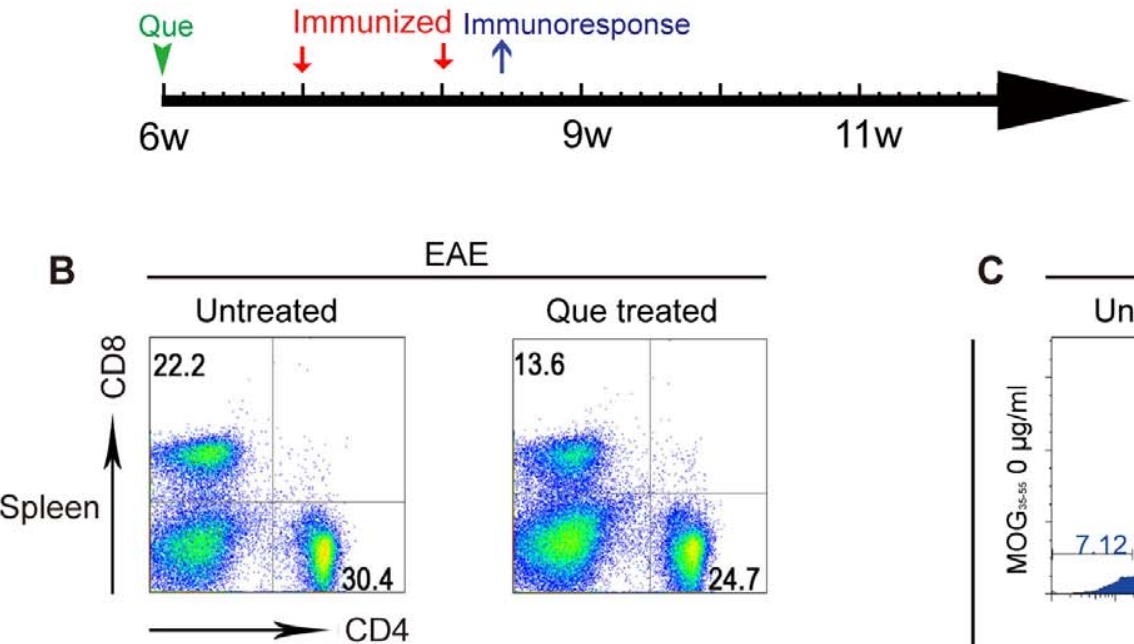

EAE
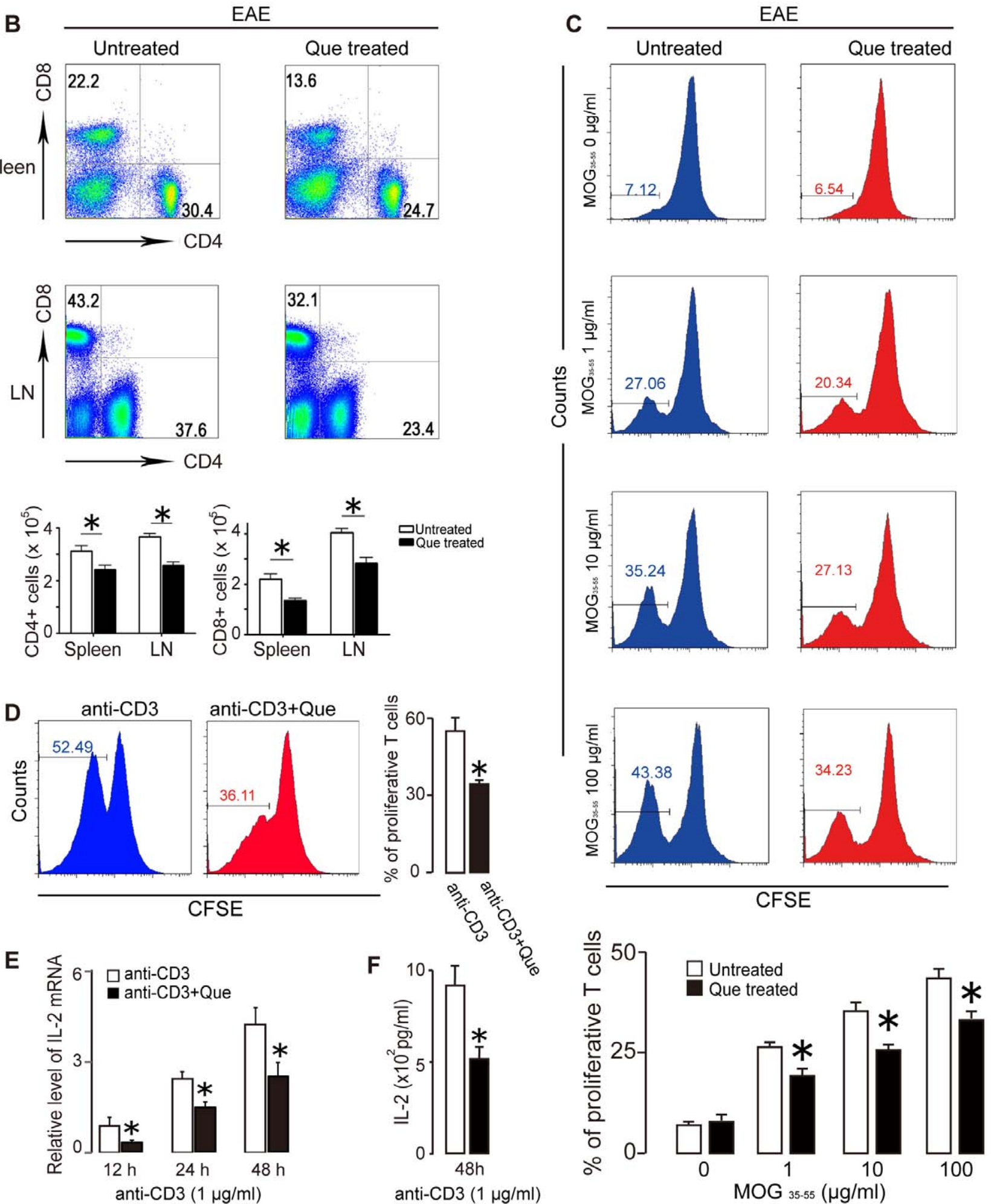
Figure 4. Que treatment decreases the number of $T$ cells in the spleen and lymph nodes and inhibites proliferation of effector $T$ cells. A. Schematic diagram displaying the time course of immunization and Que pre-treatment. B. $\mathrm{CD} 4^{+} / \mathrm{CD}^{+}$cells in the spleen and lymph nodes were quantified by FACs after Que treatment. The scatter plot (lower panel) shows the number of $\mathrm{CD} 4^{+} / \mathrm{CD}^{+}$cells per million cells. In both cases, the number of cells was decreased upon Que treatment. C. Mononuclear cells isolated from the spleens of mice were immunized with MOG $_{35-55}$ peptide with or without Que treatment, and were stimulated with $0,1,10$ or $100 \mu \mathrm{g} / \mathrm{ml}$ of MOG35-55 peptide. The proliferation was significantly suppressed assayed by CFSE. D. Effector T cells $\left(\mathrm{CD}^{+} \mathrm{CD}_{25}{ }^{-}\right)$isolated from spleen of naïve mice are labeled with CFSE. After stimulation with anti-CD3, dilution of CFSE shows that $\mathrm{CD} 4{ }^{+} \mathrm{CD} 25^{-}$populations proliferated much less vigorously after Que treatment than vehicle. E-F. IL-2 expression in effector T cells is decreased after Que-treatment assayed by either real-time PCR (E) or ELISA (F). Data represent mean \pm SEM of five independent experiments ( $N=5$, *, $p<0.05)$.

doi:10.1371/journal.pone.0042746.g004

days after immunization. Moreover, $\mathrm{CC}^{+}{ }^{+}$oligodendrocytes $(\mathrm{OLs})$ were dramatically decreased in the EAE mice with or without Que treatment as compared to unimmunized animals, while these cells were more conserved in the Que treatment group (Fig. 2C). Ki67 was used to detect the proliferative oligodendrocyte precursors (OPCs) $\left(\mathrm{NG}^{+}\right)$and a number of $\mathrm{Ki}^{+} 7^{+} / \mathrm{NG} 2^{+}$cells were seen distributed throughout the white matter in the EAE model (Figure S2). Such cells were also detected after Que treatment, however likely, to a lesser extent (Figure S2), while such proliferative OPCs were rare in normal controls. Together, these results indicate that Que treatment protects the spinal cord from demyelination and loss of OLs in EAE mouse models and OPG proliferation is a prominent response to demyelination.

Que Reduces Infiltration of T Cells in the EAE Spinal Cord

Since myelin-specific effector $\mathrm{T}$ cells migrate into CNS and initiate demyelination [4], dose the treatment of Que affect the accumulation of $\mathrm{T}$ cells in the spinal cord of EAE model? The immunohistochemical results indicate that both $\mathrm{CD}^{+}$and $\mathrm{CD} 4^{+}$ $\mathrm{T}$ cells were barely detected in unimmunized mice and control (Fig. 3A, E), while a large number of $\mathrm{CD}^{+} \mathrm{T}$ cells were found to accumulate in the spinal cord of the EAE model (Fig. 3B), especially distributed throughout the white matter. These cells were significantly decreased in the spinal cord of the Que-treated group (Fig. 3C-D). The $\mathrm{CD}^{+} \mathrm{T}$ cells were also observed infiltrated into spinal cord (Fig. 3F) and were reduced after Que treatment (Fig. 3F-H) with the exception that the number of CD8+ $\mathrm{T}$ cells was much fewer than that of $\mathrm{CD} 4^{+}$cells.

\section{Que Attenuates $\mathrm{MOG}_{35-55}$-specific Immune Response and Inhibits Effector T Cell Proliferation}

The myelin-specific effector $\mathrm{T}$ cells are generated in periphery lymph organs. To confirm the reduction of $\mathrm{CD}^{+}$and $\mathrm{CD}^{+} \mathrm{T}$ cells found in the spinal cord and to reveal their source, we detected the $\mathrm{CD} 4^{+} / \mathrm{CD}^{+} \mathrm{T}$ cell numbers from spleen and lymph nodes using FACS 10 days after immunization with Que pretreatment for 1 week (Fig. 4A) and found that Que treatment resulted in reduced numbers of $\mathrm{CD}^{+}$and $\mathrm{CD}^{+} \mathrm{T}$ cells as compared with untreated EAE mice (Fig. 4B). To test whether Que attenuated $\mathrm{MOG}_{35-55}$-specific $\mathrm{T}$ cells generation, we measured the in vitro stimulated proliferation of $\mathrm{T}$ cells isolated from spleens 10 days after $\mathrm{MOG}_{35-55}$ immunization, with or without Que pre-treatment. The results show that $\mathrm{MOG}_{35-55^{-}}$ stimatuating proliferation of $\mathrm{T}$ cells from primed mice spleen were significantly decreased in Que treated mice (Fig. 4G).

To further examine whether the reduction of T cells comprise a non-specific proliferation inhibitive effect by Que, we isolated $\mathrm{CD} 4^{+} \mathrm{CD} 25^{-} \mathrm{T}$ cells (effector $\mathrm{T}$ cells) from spleen of naïve C57BL/6 mice and stimulated the cells with anti-CD3 Ab with/ without Que treatment, dilution of CFSE results exhibit that $\mathrm{CD} 4^{+} \mathrm{CD} 25^{-} \mathrm{T}$ cells from Que treatment proliferated much less vigorously than that from untreated EAE mice (Fig. 4D). Additionally, Que decrease the expression IL-2, a potent $\mathrm{T}$ cell growth factor, assayed by either real-time PCR (Fig. 4E) or ELISA (Fig. 4F). Together, these results indicate Que can either attenuate $\mathrm{MOG}_{35-55}$-specific immune response or inhibit effector $\mathrm{T}$ cell proliferation.

\section{Que Diminishes Accumulation of $\mathrm{CD}^{+} \mathrm{T}$ Cells that Reduces OLs Loss and Demyelination in EAE Spinal Cords}

As the infiltrated $\mathrm{T}$ cells initiate OLs damage and local immunoresponse, dose reduced $\mathrm{CD}^{+} \mathrm{T}$ cells rescue demyelination and protect OLs from loss? The double labeling experiments with $\mathrm{CC1} / \mathrm{MBP}$ and $\mathrm{CD} 4$ showed $\mathrm{CD}^{+}{ }^{+}$cells were abundantly distributed in the white matter upon EAE induction (Fig. 5A,C, arrowhead), and were specifically localized to demyelination lesions (Fig. 5A), while $\mathrm{CG}^{+}$OLs were absent from these areas (Fig. 5C). These observations suggest that the accumulation of $\mathrm{CD}^{+}$cells could lead to loss of OLs and demyelination. Treatment of Que, however, decreased the number of infiltrated $\mathrm{CD}^{+}$cells, with a subsequent increase in the number of $\mathrm{CCl}^{+}$ OLs (Fig. 5D), observed adjacent to a single appearing $\mathrm{CD} 4^{+} \mathrm{T}$ cell, accompanied with normal myelin staining (Fig. 5B).

\section{Que Inhibits Activation of Microglia and Astrocytes in EAE Spinal Cord}

As local immunoresponse is initially triggered by infiltrated $\mathrm{T}$ cells and contributed to the neuronal damage and demyelination, we observed $\mathrm{CD} 68^{+}$and $\mathrm{CD} 11 \mathrm{~b}^{+}$cells which represent activated microglia/macrophages, were extensively populated throughout the spinal cord in the EAE model and were significantly diminished by Que treatment (Fig. 6A,B). Similarly, more activated $\mathrm{GFAP}^{+}$astrocytes were found in the spinal cord of EAE mice as compared to normal controls as well as Que treated EAE mice, indicating that the activation of microglia and astroctyes in EAE had been attenuated by Que treatment (Fig. 6C).

\section{Discussion}

In the present study, C57BL/6 mice immunized with $\mathrm{MOG}_{35-}$ 55 peptide display the disease symptoms associated with demyelination, glial activation, and $\mathrm{CD}^{+} \mathrm{T}$ cell infiltration in the spinal cord. These findings indicate that our EAE models are similar with previous reports and mimic the onset of MS, the T cell mediated autoimmune demyelinating disease [18]. Our study demonstrates the beneficial effects of the administration of Que, a current antipsychotic drug, on the MOG-induced EAE model. Specifically, Que treatment delays the onset, prevents deterioration of EAE symptoms, prevents demyelination, and inhibits generation of effector $\mathrm{T}$ cells in peripheral lymphatic organs that may results in reducing local inflammation in the spinal cord.

It has been previously reported that Que is protective against myelin breakdown in the cupriozne-induced demyelination model by up-regulation of SOD activity [16]. Our present observations provide further evidence to demonstrate the beneficial effects of Que on myelin forming OLs in EAE spinal cord (Fig 2, 5). Enhanced MBP expression and the number of $\mathrm{CCl}^{+}$mature OLs 

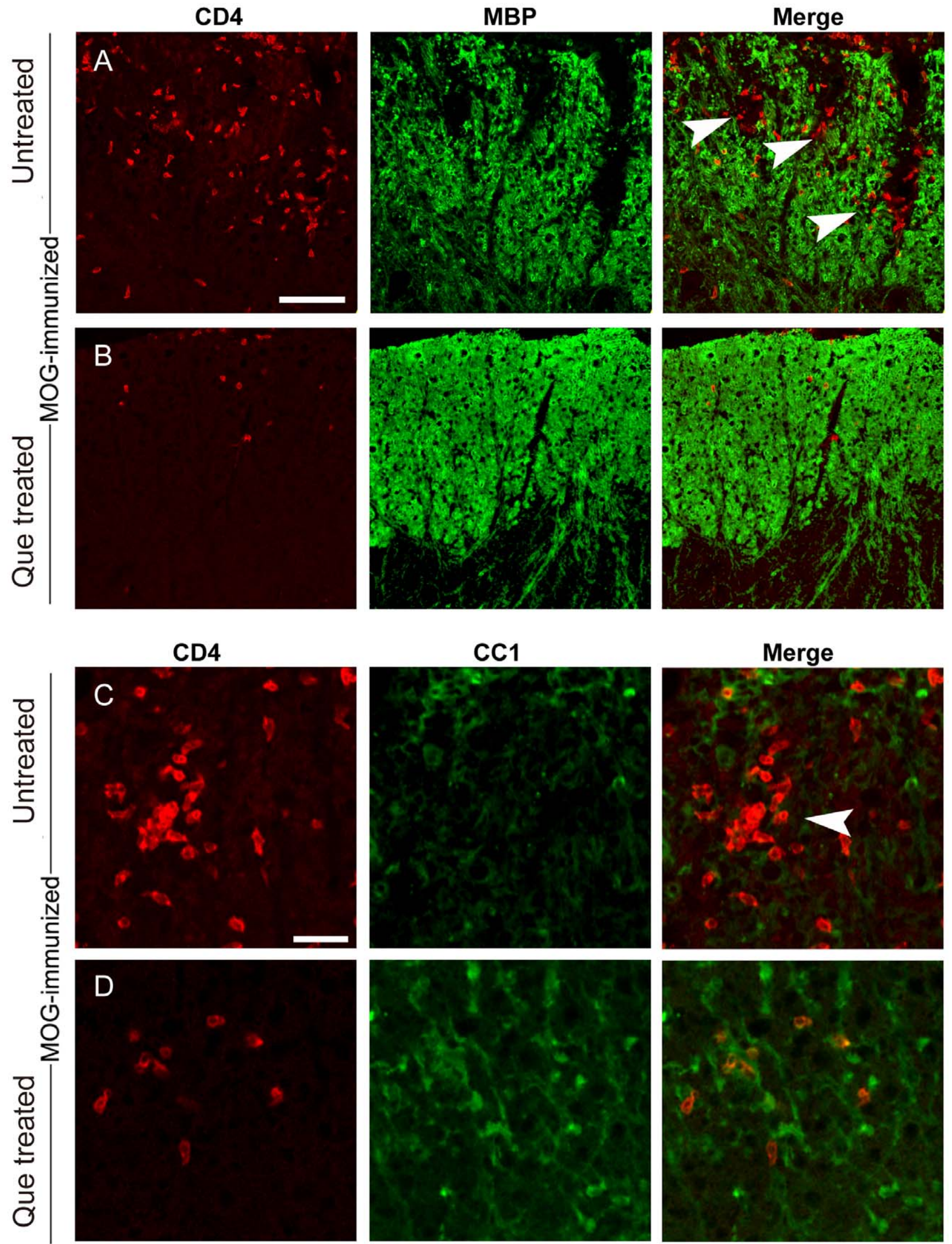

Figure 5. Que decreases the number of infiltrating $T$ cells and protects mice from demyelination and OLs loss. Micrographs display the number of infiltrating $C D 4^{+}$cells (red, A-D), MBP expression (Green, A,B) and oligodendrocytes $\left(C C 1^{+}\right.$, Green, $\mathbf{C , D}$ ) in the spinal cord of the EAE model with/without Que treatment. A-D: Clusters of infiltrating CD4 ${ }^{+}$cells are observed in the spinal cord without Que treatment $(\mathbf{A}, \mathbf{C})$ and, surrounding these areas, demyelination is present (arrowheads, $\mathbf{A})$ and is devoid of oligodendrocytes $\left(\mathrm{CC}^{+}{ }^{+}\right.$, arrowheads, C); Que treatment decreases 
$\mathrm{CD} 4^{+}$cell infiltration $(\mathbf{B}, \mathbf{D})$ and more abundant myelin segements $\left(\mathrm{MBP}^{+}, \mathbf{B}\right)$ and oligodendrocytes $\left(\mathrm{CC} 1^{+}, \mathbf{D}\right)$ are observed surrounding individual $\mathrm{CD}^{+}$cells. Scale bar $\mathbf{A}-\mathbf{B}=0.1 \mathrm{~mm} ; \mathbf{C}-\mathbf{D}=80 \mu \mathrm{m}$.

doi:10.1371/journal.pone.0042746.g005

found in the Que treated mice suggest that Que may protect OLs from undergoing cell death, consistent with the previous findings, which report that Que may alleviate oxidative stress, neutralize free radicals or modulate the expression and localization of the pro- and anti-apoptotic genes $\mathrm{Bax}$ and $\mathrm{Bcl}-\mathrm{X}(\mathrm{l} / \mathrm{s}) \quad[12,15]$. Nevertheless, we did not examine cell apoptosis in this case and cannot provide direct evidence to confirm this proposed mechanism in the current study. In trying to address the effect of Que on OL lineage, our present results demonstrate that the proliferation of NG2 positive OPCs were present in EAE mice with/without Que treatment (Figure S2), one of the prominent responses to demyelination [19]. Thus Que displays a neuroprotective capacity in EAE mice. On the other hand, Que may promote OPCs differentiate into myelin-formating OLs, especially when considering the increased number of $\mathrm{CCl}^{+}$mature OLs after Que treatment (Fig. 2, 5), combined with the previous observation that Que facilitates the maturation of newly formed OLs in cultures [14]. Given that the differentiation block of OPCs as a cause for failure of remyelination in chronic MS [20], our data suggests that the therapeutic effect of Que on EAE may be due to, at least in part, the capacity to promote OPCs differentiation into mature OLs that enhances remyelination in EAE model, similar to certain
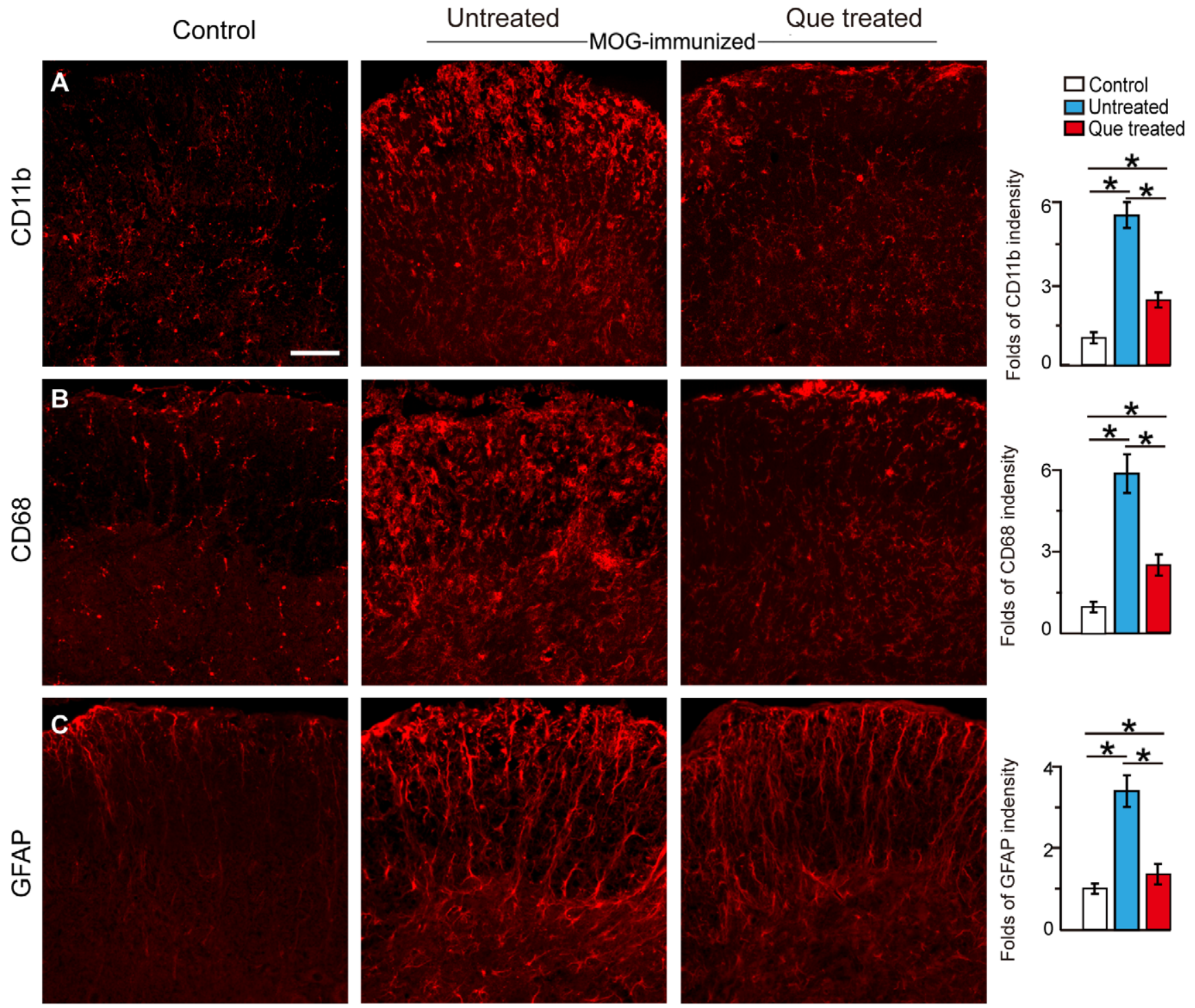

Figure 6. Que inhibits the activation of microglia/macrophages and astrocytes in spinal cord. Immunofluorescent staining with antiCD68 and CD11b antibodies to demonstrate the number of microglia/macrophages in the EAE model with/without Que treatment, A. Numerous $\mathrm{CD} 11 \mathrm{~b}^{+}$microglia/macrophages were observed in the EAE model which were greatly decreased after Que treatment. B. CD68 staining displayed a similar staining pattern after Que treatment. C. GFAP immunofluorescent staining displayed an increase in reactive astrocytes in the spinal cord, which was greatly decreased after Que treatment $\left(\mathrm{N}=5,{ }^{*}, p<0.05\right)$. Quantification of the immunostaining is present in the bar graphs. Scale bar A$\mathrm{C}=0.2 \mathrm{~mm}$.

doi:10.1371/journal.pone.0042746.g006 
growth factors such as insulin-like growth factor 1 (IGF-1) or T3 $[21,22]$ which have been shown to promote remyelination efficiency in EAE even the precise molecular mechanisms remain unclear.

Regarding the pathophysiology of MS and/or EAE, it has been shown that active $\mathrm{CD} 4^{+} \mathrm{T}$ cells may trigger local neuroinflammation and further induce demyelination [3]. In our study, we provide morphological evidences demonstrating that $\mathrm{CD} 4^{+} \mathrm{T}$ cells are harmful for OLs lineage as neither OLs nor MBP expression can be detected around the lesion area with the accumulation $\mathrm{CD}^{+} \mathrm{T}$ cells (Fig. 3-5). Que treatment, however, reduces the infiltration of $\mathrm{CD}^{+} \mathrm{T}$ as well as the activation of microglial and astroglial in the spinal cord of EAE mice (Fig. 6). Although the inhibitory effect of Que on microglial activation has been described in the cuprizone induced demyelination mouse model [16], our current data provide further evidence to suggest that Que has the potential capacity to reduce microglial and astroglial activation, and decrease myelin-specific $\mathrm{T}$ cells infiltration.

In respect with the decrease of $\mathrm{T}$ cell infiltration, does it due to an inhibited local immune response in CNS or alternatively a decreased effector $\mathrm{T}$ cell generation in peripheral immune organs? As in MS and EAE, the generation of myelin protein-specific T cells has been considered as a hallmark that triggering local inflammation and demyelination. A number of drugs, such as glatiramer acetate (GA) or lithium have been found to decrease the generation of myelin protein-specific $\mathrm{T}$ cells that contribute to their therapeutic effect on MS or EAE [10,23]. In our study, we demonstrate that both $\mathrm{CD} 4^{+}$and $\mathrm{CD}^{+}$cell populations decreased in peripheral immune organs after Que treatment (Fig. 4B). Moreover, we found that Que inhibited either MOG $_{35-55}$-specific immunoresponse or $\mathrm{CD} 3 \mathrm{Ab}$ induced non-specific $\mathrm{T}$ cell proliferation and the transcriptional level of IL-2 (Fig. 4C-F), a crucial cytokine to initiate and maintain $\mathrm{T}$ cell proliferation $[24,25]$. Therefore, the decreased infiltration of $\mathrm{CD}^{+} / \mathrm{CD}^{+} \mathrm{T}$ cells found in spinal cord after Que treatment seems mainly due to the immnunomodulatory role of Que that decreases effector $\mathrm{T}$ cell generation and inhibits local immunoresponsiveness. This data reveals for the first time, that Que displays an efficacy of modulating immunoreaction and may potentially be used in the treatment of other autoimmune disease beyond MS.

As an atypical antipsychotic drug, what molecular mechanisms probably underlie the immunomodulatory effects? Recently, several neurotransmitters have been considered as potent immune-modulators. For instance, serotoninergic receptors are expressed by a broad range of inflammatory cell types, including dendritic cells, helper T cells, cytotoxin T cells and so on [26,27]. 5-HT can induce Ag-specific Thl and cytotoxin T cell proliferation via 5-HT receptor 2 and blockade of 5-HT receptor 1 attenuates deterioration of EAE model [26,27]. Similarly, histamine increases both ConA-dependent non-specific and TCRmediated Ag-specific T cell proliferation via histamine receptor 2 and 1 respectively $[28,29]$. Giving that Que acts as an antagonist of multiple neurotransmitter receptors, including serotonin 5$\mathrm{HT}(1 \mathrm{~A}), \quad 5-\mathrm{HT}(2 \mathrm{~A})$, dopamine $\mathrm{D}(1), \mathrm{D}(2)$, histamine $\mathrm{H}(1)$, adrenergic alpha(1) and alpha(2) receptors [30,31], it is possible that the antagonistic effect of Que on 5-HT receptors or other a

\section{References}

1. Bitsch A, Schuchardt J, Bunkowski S, Kuhlmann T, Bruck W (2000) Acute axonal injury in multiple sclerosis. Correlation with demyelination and inflammation. Brain 123: 1174-83.

2. Dutta R, Chang A, Doud MK, Kidd GJ, Ribaudo MV, et al. (2011) Demyelination causes synaptic alterations in hippocampi from multiple sclerosis patients. Ann Neurol 69: 445-54. broad range receptors may contribute to its immune-modulatory effect, especially the inhibition of effector $\mathrm{T}$ cell proliferation. To substantiate this speculation, extensive immunological experiments are required in further studies.

The finding that the beneficial efficacy of Que on EAE mice is particularly relevant to clinical studies, in that some patients with MS also display a variety of psychiatric symptoms, including depression or cognitive dysfunction [32], and some antipsychotic drugs, or antidepressants have been used to treat these symptoms. For example, fluoxetine, an antidepressant, has been effective in reducing lesions in relapsing MS patients [33]. Morever, oligodendrocyte dysfunction or demyelination has recently been implicated in the pathophysiology of schizophrenia, bipolar disorder and major depression [12,34,35]. Interestingly, all three disorders display major overlapping domains in their transcription profiles, especially genes involved in energy metabolism, inflammation and myelination [36]. Therefore, our present observations may support the notion that some psychiatric disorders such as schizophrenia, bipolar disorder or major depression may share similarities in disease mechanism with MS.

In conclusion, this study demonstrates that the atypical antipsychotic drug Que exerts immunomodulatory role and prevent mice from deterioration of EAE symptoms and demyelination. The novel effect of Que described here may lead to more effective strategies for treating not only schizophrenia, but other autoimmune diseases such as MS, and also provides new insight into pathogenesis of schizophrenia and related psychiatric disorders.

\section{Supporting Information}

Figure S1 Pre-treatment of Que delays the onset of EAE and relieves the symptoms. Que pre-treatment $(\mathrm{N}=10)$ was initiated 7 days before immunization, delays the onset of EAE and relieves the symptoms as compared with vehicle $(\mathrm{N}=10)(p<0.05)$. (TIF)

Figure S2 Que decreases the proliferation of OPGs in spinal cord. Ki67 (red) and NG2 (green) double immunostaining identifies the proliferating OPCs in the spinal cord. A: Ki67/NG2 double-labeled cells (arrows) are often observed in the EAE model without Que treatment, and such cells are also present in the EAE model with Que treatment (A'), however, cell numbers are diminished as compared to EAE models without Que treatment, displayed in the magnified panels (B-E, B'-E'). Scale bar, A$\mathrm{F}=0.2 \mathrm{~mm}$.

(TIF)

Table S1 The antibody information.

(DOC)

\section{Author Contributions}

Conceived and designed the experiments: LX FM JMK XML. Performed the experiments: FM SG YTH LYW HKW JQN. Analyzed the data: FM SG LX. Contributed reagents/materials/analysis tools: FM SG LX YW. Wrote the paper: LX FM JMK XML SG.

3. Fletcher JM, Lalor SJ, Sweeney CM, Tubridy N, Mills KH (2010) T cells in multiple sclerosis and experimental autoimmune encephalomyelitis. Clin Exp Immunol 162: 1-11.

4. Jones RE, Bourdette DN, Offner H, Vandenbark AA (1990) Myelin basic protein-specific $\mathrm{T}$ cells induce demyelinating experimental autoimmune encephalomyelitis in Buffalo rats. J Neuroimmunol, 30: 61-9. 
5. Liu L, Darnall L, Hu T, Choi K, Lane TE, et al. (2010) Myelin repair is accelerated by inactivating CXCR2 on nonhematopoietic cells. J Neurosci 30: 9074-83.

6. Gordon D, Pavlovska G, Uney JB, Wraith DC, Scolding NJ (2010) Human mesenchymal stem cells infiltrate the spinal cord, reduce demyelination, and localize to white matter lesions in experimental autoimmune encephalomyelitis. J Neuropathol Exp Neurol 69: 1087-95.

7. Kanwar JR, Kanwar RK, Krissansen GW (2004) Simultaneous neuroprotection and blockade of inflammation reverses autoimmune encephalomyelitis. Brain 127: $1313-31$

8. Makar TK, Trisler D, Bever CT, Goolsby JE, Sura KT, et al. (2008) Stem cell based delivery of IFN-beta reduces relapses in experimental autoimmune encephalomyelitis. J Neuroimmunol 196: 67-81.

9. Constantin G, Marconi S, Rossi B, Angiari S, Calderan L, et al. (2009) Adiposederived mesenchymal stem cells ameliorate chronic experimental autoimmune encephalomyelitis. Stem Cells 27: 2624-35.

10. Aharoni R, Herschkovitz A, Eilam R (2008) Demyelination arrest and remyelination induced by glatiramer acetate treatment of experimental autoimmune encephalomyelitis. Proc Natl Acad Sci USA 105: 11358-63.

11. Trapp BD, Nave KA (2008) Multiple sclerosis: an immune or neurodegenerative disorder? Annu Rev Neurosci 31: 247-69.

12. Wei Z, Mousseau DD, Richardson JS, Dyck LE, Li XM (2008) Atypical antipsychotics attenuate neurotoxicity of beta-amyloid(25-35) by modulating Bax and Bcl-X $(1 / \mathrm{s})$ expression and localization. J Neurosci Res 74: 942-7.

13. Kerns D, Vong GS, Barley K, Dracheva S, Katsel P, et al. (2010) Gene expression abnormalities and oligodendrocyte deficits in the internal capsule in schizophrenia. Schizophr Res 120: 150-8.

14. Xiao L, Xu H, Zhang Y, Wei Z, He J, et al. (2008) Quetiapine facilitates oligodendrocyte development and prevents mice from myelin breakdown and behavioral changes. Mol Psychiatry 13: 697-708.

15. Xu H, Wang H, Zhuang L, Yan B, Yu Y, et al. (2008) Demonstration of an antioxidative stress mechanism of quetiapine: implications for the treatment of Alzheimer's disease. FEBS J 275: 3718-28.

16. Zhang Y, Xu H, Jiang W, Xiao L, Yan B, et al. (2008) Quetiapine alleviates the cuprizone-induced white matter pathology in the brain of C57BL/6 mouse. Schizophr Res 106: 182-91.

17. Bian Q Kato T, Monji A, Hashioka S, Mizoguchi Y, et al. (2008) The effect of atypical antipsychotics, perospirone, ziprasidone and quetiapine on microglial activation induced by interferon-gamma. Prog Neuropsychopharmacol Biol Psychiatry $32: 42-8$.

18. Stromnes IM, Goverman JM (2006) Active induction of experimental allergic encephalomyelitis. Nat Protoc 1: 1810-9.

19. Rasmussen S, Imitola J, Ayuso-Sacido A, Wang Y, Starossom SC, et al. (2011) Reversible neural stem cell niche dysfunction in a model of multiple sclerosis. Ann Neurol 69: 878-91.

20. Kuhlmann T, Miron V, Gui Q, Wegner C, Antel J, et al. (2008) Differentiation block of oligodendroglial progenitor cells as a cause for remyelination failure in chronic multiple sclerosis. Brain 131: 1749-58.
21. Franco PG, Silvestroff L, Soto EF, Pasquini JM (2008) Thyroid hormones promote differentiation of oligodendrocyte progenitor cells and improve remyelination after cuprizone-induced demyelination. Exp Neurol 212: 458-67.

22. Mason JL, Ye P, Suzuki K, D’Ercole AJ, Matsushima GK (2000) Insulin-like growth factor-1 inhibits mature oligodendrocyte apoptosis during primary demyelination. J Neurosci 20: 5703-8.

23. De SP, Axtell RC, Raman C, Roth KA, Alessi DR, et al (2008) Lithium prevents and ameliorates experimental autoimmune encephalomyelitis. J Immunol 181: $338-45$.

24. Chang JF, Thomas CA III, Kung JT (1991) Mitogen-induced IL-2 production and proliferation at defined stages of T helper cell development. J Immunol 147: 860-6.

25. Maruo S, Toyo-oka K, Oh-hora M, Tai XG, Iwata H, et al. (1996) IL-12 produced by antigen-presenting cells induces IL-2-independent proliferation of T helper cell clones. J Immunol 156: 1748-55.

26. Inoue M, Okazaki T, Kitazono T, Mizushima M, Omata M, et al. (2011) Regulation of antigen-specific CTL and Th1 cell activation through 5Hydroxytryptamine 2A receptor. Int Immunopharmacol 11: 67-73.

27. Freire-Garabal M, Nunez MJ, Balboa J, Garcia-Vallejo LA, Argibay S, et al. (2003) Administration of the 5-hydroxytryptamine(1A) receptor antagonist WAY100635 suppresses acute experimental allergic encephalomyelitis in Lewis rats. Neurosci Lett342: 33-6.

28. Nakane H, Sonobe Y, Watanabe T, Nakano K (2004) Histamine its novel role as an endogenous regulator of Con A-dependent T cell proliferation. Inflamm Res 53: $324-8$.

29. Noubade R, Milligan G, Zachary JF, Blankenhorn EP, del Rio R, et al. (2007) Histamine receptor $\mathrm{H} 1$ is required for TCR-mediated p38 MAPK activation and optimal IFN-gamma production in mice. J Clin Invest 117: 3507-18.

30. Jones HM, Travis MJ, Mulligan R, D Visvikis, S Gacinovic, et al. (2000) In vivo serotonin 5-HT(2A) receptor occupancy and quetiapine. Am J Psychiatry 157: 148.

31. Ray LA, Heydari A, Zorick T (2010) Quetiapine for the treatment of alcoholism: scientific rationale and review of the literature. Drug Alcohol Rev 29: 568-75.

32. Janardhan V, Bakshi R (2002) Quality of life in patients with multiple sclerosis: the impact of fatigue and depression. J Neurol Sci 205: 51-8.

33. Mostert JP, Admiraal-Behloul F, Hoogduin JM, Luyendijk J, Heersema DJ, et al. (2008) Effects of fluoxetine on disease activity in relapsing multiple sclerosis: a double-blind, placebo-controlled, exploratory study. J Neurol Neurosurg Psychiatry 79: 1027-31.

34. Kerns D, Vong GS, Barley K, Dracheva S, Katsel P, et al. (2010) Gene expression abnormalities and oligodendrocyte deficits in the internal capsule in schizophrenia. Schizophr Res 120: 150-8.

35. Aston C, Jiang L, Sokolov BP (2005) Transcriptional profiling reveals evidence for signaling and oligodendroglial abnormalities in the temporal cortex from patients with major depressive disorder. Mol Psychiatry 10: 309-22.

36. Konradi C, Sillivan SE, Clay HB (2012) Mitochondria, oligodendrocytes and inflammation in bipolar disorder: evidence from transcriptome studies points to intriguing parallels with multiple sclerosis. Neurobiol Dis 45: 37-47. 